\title{
Lactancia materna y aumento de peso en el primer año de vida
}

\author{
Ise López, ' Carmen Cabiol, 2 Sarn Arcuch, ${ }^{2}$ Elians Rivera ${ }^{2}$ y Sergio Varas'
}

\author{
ABSTRACT
}

\begin{abstract}
This is the first part of a study of 207 infiants 0 to 12 months of age. we tried to prove that breast-feeding contributes positively to gro wth, measured according to gain in weight and stature, and that in reduces morbidity caused by diarrhen and malnulrition during the first year of life.

All the children came frora low-income families, and were beneficiaries of one of the National Health Servipe's outpatient climics in Sentiago.

Fifty per cent of the group was found to have been exclusively breast fed for up 103 months. Slightly over $1 / 3$ was still being breast fed at 6 montbs, only $15 \%$ at 10 months, and more than $9 \%$ at 12 munth of age. If breast-feeding plus formula is considered, $50 \%$ wat being partly bresst fed at 8 month, $36 \%$ at 10 months, and $25 \%$ at 12 month.

An important difference was observed when taking birth weight into accuunt. Fifty per cent of infants whose birth weight was Irom $2500 \mathrm{gr}$. to $3490 \mathrm{gr}$. were breast fed for only $31 / 2$ months, while the same percentsge of those weighing bet ween $3500 \mathrm{gr}$. and $4190 \mathrm{gr}$. were breast fed for 5 months.

Monthly weight gain for breast fed infants was significonlly higher than that for bottle fed infants during the first nine monıhs. However, even for breast fed babjes, weight increase after the 8 th month was lower than that indicaled in sturt's 50 percentile.
\end{abstract}

"La manera más fisiológica de alimentar a un nin̄o en los primeros meses es con leche de su madre. Las razones que pueden invocarse a favor de este tipo de alimentación son numerosas: la leche humana posee la composición adecuada al organismo al que está destinada; es el alimento más higiénico, pues pasa directamente del pecho materno a la boca del niño; no está, por razón expuesto a fraude o contaminación, es el más económico y sirve al parecer para establecer vínculos afectivos entre la madre y el hijo". I

Sobre el periodo injcial de amamantamiento no hay unanimidad entre las opiniones de los especialistas, sin embargo, es evidente que en Chile las madres han Llegado a límites muy bajos. Muchos estudios así lo han demostrado. En 600 casos pertenecientes a consultorios urbanos y rurales del Area Hospitaloria Norte de Santiago, estudiados en 1968, se encontró que a los 2 meses de vida el $50 \%$ de los niños ya estaban recibiendo otros tjpos de leche. ${ }^{1}$ En otro estudio realizado en 1.050 niños de diferentes estratos sociales, entre 1975 y 1976, se determinó que la mitad de los niños recibian leche matema hasta los 3 meses. 2

Se afirma que, "en Chile, como en otros paises latinoamericanos una de las causas más importantes de la desnutrición es la brevedad e insuficiencia de Ia lactancia materna.' En consecuencia, ésta debe estjmularse y tratar de prolongarse con el fin no sólo de evitar la carencia alimentaria sino también las in-

\footnotetext{
${ }^{1}$ Acadéraicos Departamento de Salud Pública y Medicina Socual. Facultad Medicina Norte, Universidad de Chile.

${ }^{2}$ Académicos Departamento Matemo lnfantil. Facultad de Medicina Sur. Universidad de Chile.
}

fecciones que en muchos casos traen como consecuencia la desnutrición. En 1979, la tasa de desnutrición de menores de 1 año controlados por el Servicio Nacional de Salud alcanzó a 13,2\%, 6 razón que puede considerarse como suficiente para continuar insistiendo en estudios que satisfacen las bondades de la leche materna como alimento lácteo durante el primer año de vida.

Este estudio pretende cuantificar los beneficios que recibe el niño menor de un año, al ser alimentado por su madre en la primera etapa de su vida, como son el crecimiento en peso y talla y evitar algunas enfermedades relacionadas con el tipo de alimentación que muchas veces conducen a la desnutrición.

\section{Hipotesis.}

Con los antecedentes disponibles se puede postular que la leche materna contribuye positivamente al crecimiento del niño en peso y talla y disminuye la taga de morbilidad por diarreas y desnutrición durante el primer ano de vida. En este primer informe se presentan los resultados observados para el aumento de peso.

\section{METODOLOGIA}

Se observó, mes a mes, durante el primer año de vida un grupo de 207 niños de la población asignada al Consultorio Nogales, perteneciente al Area de Salud Central de Santiago, y que se inscribieron en este establecimiento en los primeros 20 dias después de necer.

Con el fin de evitar la interferencia de algunas va- 
riables los niños del estudio debían cumplir los siguientes requigitos:

a) Un peso al nacer de $2.500 \mathrm{~g}$ o más;

b) Haber nacido de parto normal;

c) No haber presentado morbilidad importante en los primeros 7 dias de vida;

d) Haber obtenido un apgar de 80 más;

e) No haber sido hospitalizado;

f) Haber tenido un período gestacional normal y no inferior a 38 semanas;

g) Que durante el embarazo la madre no haya presentado algín tipo de morbilidad importante como diabetes, sifilis, pre-eclampsia, ictericia gravídica y Rh (-).

Según normas establecidas por el Ministerio de Salud, los lactantes deben acudir al control una vez a mes durante el primer semestre y luego a $\operatorname{los} 8,10$ y 12 meses. En cada control se registró el peso, la talla, el tipo de alimentación láctea al momento del control, cambios introducidos en la alimentación entre los controles, cuadros mórbidos presentados en el mes. Esta información se extrajo desde la historia clínica y fue vaciada en formularios especialmente diseñados para este objeto.

Al término del período de seguimiento, el $44 \%$ de los niños en estudio fue visitado, en sus domicilios, con el fin de recoger información socioeconómica del grupo.

\section{RESULTADOS}

Caracteristicas del grupo en estudio:-Este incluye $\mathbf{8 4}$ hombres y $\mathbf{1 2 0}$ mujeres. Según el peso al nacer, cerca de la mitad pesaron entre 3.000 y 3.490 g y el resto se distribuyó casi simétricamente entre $\log 2.500$ y 2.990 g y 3.500 y $4.000 \mathrm{~g}$, respectivamente. La talla varió entre 44 y $55 \mathrm{~cm}$, concentrándose el $74 \%$ entre 48 y 51 centímetros.

Las madres de estos niños en un $57 \%$ tenian entre 20 y 29 años. El resto se distribuyó simétricamente en menos de 20 años y de 30 a 39 años.

El $22 \%$ presentaba un estado civil inestable con un nivel de escolaridad bajo, poco más de la mitad, un $52 \%$, djce haber aprobado entre 4 y 6 años de escuela; a éstos se pueden agregar un $3,6 \%$ con menos de 4 años o sin escolaridad.

La mayoría de estos niños habitan casas con un abastecimiento de agua potable adecuado y buen sistema de eliminación de excretas. Sin embargo, éstas se acompañan de un alto grado de hacinamientos en viviendas de mala calidad (el $57 \%$ de las familias duermen más de dos personas por cama; el $63 \%$ habitan más de 3 personas por pieza y $44 \%$ de los niños forman parte de familia de $6 \mathrm{y}$ más miembros).

Por lo tanto, puede afirmarse que este grupo de niños pertenece a un estrato socioeconómico bajo.

\section{DURACION DEL PERIODO \\ DE AMAMANTAMIENTO}

En cada mes de vida se averiguó el tipo de leche que recibía cada niño. Los resultados se presentan en la Tabla 1.

Tabla N. ${ }^{\circ} 1$

Porcentajes de niños según tipo de alimentación láctea en cada mes de vida

\begin{tabular}{|c|c|c|c|c|}
\hline \multirow[t]{2}{*}{ Edad } & \multirow{2}{*}{$\frac{\text { Natural }}{\%}$} & \multirow{2}{*}{$\frac{\text { Mixta }}{\%}$} & \multirow{2}{*}{$\frac{\text { Artificial }}{\%}$} & \multirow[t]{2}{*}{ Total } \\
\hline & & & & \\
\hline $15 \mathrm{ds}$. & 91,8 & 6,8 & 1,4 & 207 \\
\hline 1 & 79,2 & 15,0 & 5,8 & 207 \\
\hline 2 & 71,1 & 18,8 & 10,1 & 207 \\
\hline 3 & $\mathbf{5 7 , 0}$ & 27,5 & 15,5 & 207 \\
\hline 4 & 47,4 & 30,9 & 21,7 & 207 \\
\hline 5 & 42,9 & 30,9 & 26,2 & 207 \\
\hline 6 & 35,9 & 27,7 & 36,4 & 206 \\
\hline 7 & 34,5 & 26,7 & 38,8 & 206 \\
\hline 8 & $26, \overline{7}$ & 22,3 & 51,0 & 206 \\
\hline 9 & 25,2 & 20,9 & $5 \mathbf{3}, 9$ & 206 \\
\hline 10 & 15,5 & 20,9 & 73,6 & 206 \\
\hline 11 & 12,6 & 20,9 & 64.5 & 206 \\
\hline 12 & 8,9 & 15,7 & 75,4 & 206 \\
\hline
\end{tabular}

Al primer mes de vida un $20 \%$ niños está recibiendo leche artificial sola o como complemento de la natural. A los 3 meses esta proporción aumenta a $43 \%$ y a los 6 meses los que sólo reciben leche materna alcanzan a un $36 \%$, proporción que disminuye notoriamente hasta llegar a los 12 meses sólo un $\mathrm{B}, 9 \%$.

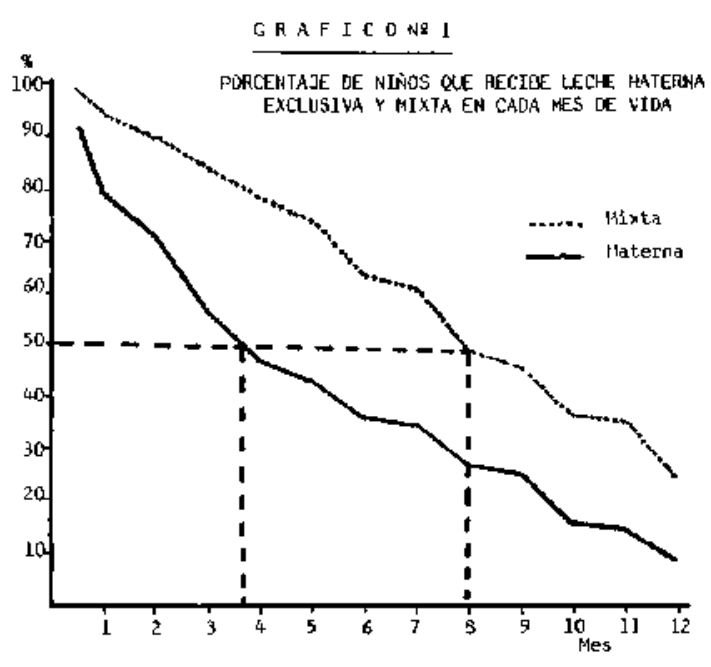


Al calcular la mediana de duración para la lactancia materna, sólo se obtienen 3 meses y 3 semanas. EI mismo indicador para niños alimentados con leche materna sola o complementada con leche artificial se logran 8 meses de duración. Significa que el $50 \%$ de los niños estudiados está recibiendo leche materna en alguna proporción hasta cumplir los 8 meses.

Queda a criterio de los especialistas juzgar en qué medida significa un beneficio para el nin̄o, al recibir leche materna en alguna cantidad.

Aunque este estudio sólo comprende 207 niños de un área de Santiago, al comparar los resultados con otros realizados con muestras más amplias, ${ }^{2}$ pero en el mismo período de tiempo, se puede concluir que los resultados, en cuanto a lactancia materna absoluta, son coincidentes. El $50 \%$ de los niños, sin considerar otras variables, no llega a los 4 meses de amamantamiento.

\section{PESO AL NACER Y DURACION DE LACTANCIA MATERNA}

Los niños fueron clasificados en tres grupos, según el peso de nacimiento y se analizó la tendencia del período de amamantamiento en cada uno de ellos. (En la categoría de 3.500 a 3.990 se incluyen 5 niños que pesaron entre 4.000 y 4.190 gramos).

Tabla N. 2

Porcentaje de nin̄os en cada tipo de alimentación láctea, según grupo de peso al nacer

\begin{tabular}{|c|c|c|c|c|c|c|c|c|c|}
\hline \multirow{2}{*}{$\begin{array}{l}\text { Edad } \\
\text { (meses) }\end{array}$} & \multicolumn{3}{|c|}{$2.500-2.900(n=58)$} & \multicolumn{3}{|c|}{$3.000-3.490(n=94)$} & \multicolumn{3}{|c|}{$3.500-3.990(n=55)$} \\
\hline & Nat. & Mixta & Art. & Nat. & Mixta & Art. & Nat. & Mixta & Art. \\
\hline 15 días & 96,6 & 1,7 & 1,7 & 91,5 & 7,4 & 1,1 & 87,3 & 10,9 & 1,8 \\
\hline 1 & 84,5 & 12,1 & 3,4 & 76,6 & 13,8 & 9,6 & 80,0 & 18,2 & 1,8 \\
\hline 2 & 74,1 & 19,0 & 6,9 & 64,9 & 20,2 & 14,9 & 78,2 & 16,4 & 5,5 \\
\hline 3 & 53,4 & 32,8 & 13,8 & 55,3 & 24,5 & 20,2 & 63,6 & 27,3 & 9,1 \\
\hline 4 & 41,4 & 36,2 & 22,4 & 44,7 & 28,7 & 26,6 & 56,4 & 30,9 & 12,7 \\
\hline 5 & 39,7 & 31,0 & 29,3 & 40,4 & 28,7 & 30,9 & 50,9 & 34,5 & 14,5 \\
\hline 6 & 31,6 & 31,6 & 36,8 & 33,0 & 26,6 & 40,4 & 45,5 & 25,5 & 29,1 \\
\hline 7 & 28,1 & 33,3 & 38,6 & 31,9 & 24,5 & 43,6 & 45,5 & 23,6 & 30,9 \\
\hline 8 & 24,6 & 19,3 & 56,1 & 25,5 & 24,5 & 50,0 & 30,9 & 21,8 & 47,3 \\
\hline 9 & 21,1 & 21,1 & 57,8 & 24,5 & 23,4 & 52,1 & 30,9 & 16,4 & $50,9^{4}$ \\
\hline 10 & 5,3 & 21,1 & 73,7 & 18,1 & 20,2 & 61,7 & 21,8 & 21,8 & 54,5 \\
\hline 11 & 5,4 & 21,4 & $73.2^{2}$ & 18.1 & 20,2 & 61,7 & 18,2 & 21,8 & 58,2 \\
\hline 12 & 5,4 & 19,6 & $73,2^{3}$ & 18,8 & 11,7 & 75,5 & 5,5 & 18,2 & 74,5 \\
\hline
\end{tabular}

$1_{n}=57 \quad{ }^{2} n=56 \quad 3_{n}=55 \quad 4_{n}=54$

Gráfico N. ${ }^{\circ} 2$

Proporción de niños que recibe leche materna en cada mes de edod según peso al nocer

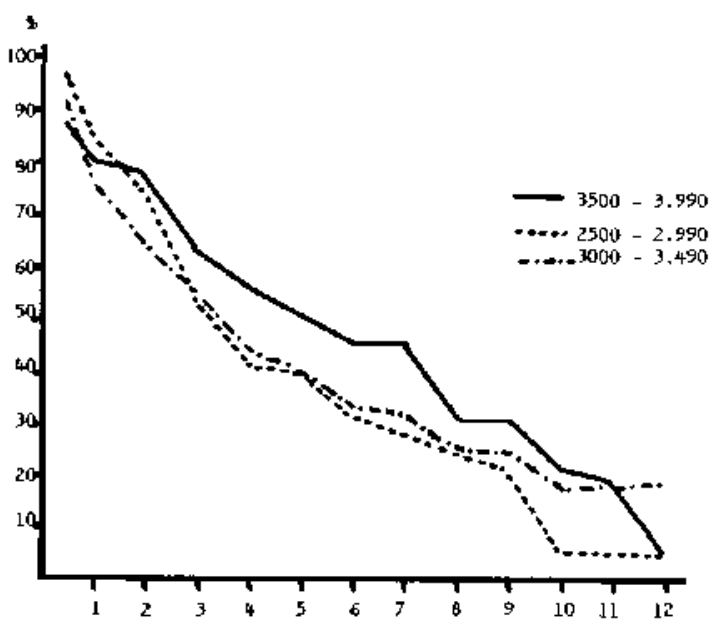

En el primer trimestre hay una disminución más importante de los grupos de menos de $3.500 \mathrm{~g}$. sólo un $55 \%$ de estos niños continúa al pecho, al cumplir 3 meses una proporción semejante del grupo de $3.500 \mathrm{~g}$ o más se observa un mes después. El comportamiento de los grupos de menos peso es muy semejante hasta el 9. ${ }^{\circ}$ mes al que alcanza cerca de la cuarta parte de los niños. A partir del 10. "mes el grupo de menos de $3.000 \mathrm{~g}$ se reduce a un $5 \%$. El grupo intermedjo, en cambio, se mantiene en un $18 \%$. Los niños de mayor peso al nacimiento disminuye en forma más lenta. EJ $45 \%$ alcanza al menos 7 meses. Dos meses más tarde se encuentra aún un $30 \%$. A partir de los 10 meses la tendencia baja hasta alcanzar al año una proporción semejante al grupo de peso inferior a 3.000 gramos.

Al calcular la mediana de duración en cada grupo, se aprecia que el $50 \%$ de los niños que pesaron menos de $3.500 \mathrm{~g}$ alcanzan como máximo 3 meses y medio, mientras que la mitad de los niños que pesaron 
entre 3.500 y $4.190 \mathrm{~g}$ legan a los 5 meses. El $50 \%$ de los que reciben leche materna exclusiva más los que reciben complemento alcanzan hasta los $\mathbf{8}$ meses.

\section{AUMENTO DE PESO}

Durante el primer año de vida, a partir de la primera quincena, el incremento de peso es siempre ascendente con un ritmo diferente entre el primer y segundo semestre. El peso teórico normal se determina sumando el peso al nacer incrementos ponderales mensuales que corresponden al $\mathbf{P}_{50}$ de Stuart. ${ }^{4,1,5}$
$600 \mathrm{~g}$ al primer mes, $900 \mathrm{~g}$ al $2 .^{\circ}$ y $3 .^{\circ} ; 600 \mathrm{~g}$. , cada mes, entre el $4 .^{\circ}$ y $6 .^{\circ} ; 500 \mathrm{~g}$ mensuales entre $7 .^{\circ}$ y $90^{\circ}$ y $300 \mathrm{~g}$ por mes entre $10 .^{\circ}$ y $12 .^{\circ}$. Lo que significa que a los 12 meses el niño debe haber aumentado $6.600 \mathrm{~g}$ su peso de nacimiento.

Los nifios se clasificaron, para cada mes de vida, según el tipo de alimentación láctea que recibieron hasta ese momento. Se calculó para cada niño la diferencia de peso entre ese momento y el nacimiento y luego se resumió para cada edad y grupo de lactancia, en un promedio de aumento. Los resuliados ae presentan en la Tabla 3.

Tabla N. ${ }^{\circ} 3$

Promedio de aumento de peso en cada mes de vida según tipo de alimentación láctea, desviación standard (s), tamaño del grupo (n)

\begin{tabular}{|c|c|c|c|c|c|c|c|c|c|c|}
\hline \multicolumn{11}{|c|}{ Tipo de lactancia } \\
\hline \multirow{2}{*}{$\begin{array}{l}\text { Edad } \\
\text { (ms) }\end{array}$} & \multirow{2}{*}{$\begin{array}{l}\text { Aumento } \\
\text { esperado } \\
\text { acumul. }\end{array}$} & \multicolumn{3}{|c|}{ Natural } & \multicolumn{3}{|c|}{ Mixta } & \multicolumn{3}{|c|}{ Artificial } \\
\hline & & $\overline{\mathbf{x}} *$ & 8 & $\mathbf{n}$ & $\overline{\mathbf{x}}$ & B & n & $\overline{\mathbf{x}}$ & 8 & $\mathbf{n}$ \\
\hline 1 & 600 & 779.1 & 357.9 & 156 & 469 & 436.9 & 29 & 513.3 & 415.? & 12 \\
\hline 2 & 1500 & 1770.9 & 535.7 & 139 & 1449.1 & 622.5 & 33 & 1067.0 & 506.5 & 20 \\
\hline 3 & 2400 & 2587.6 & 622.8 & 109 & 2299.1 & 758.0 & 53 & 1922.3 & 802.8 & 26 \\
\hline 4 & 3000 & 3314.8 & 696.6 & 90 & 3040.7 & 768.0 & 63 & 2634.6 & 948.9 & 42 \\
\hline 5 & 3600 & 3832.7 & 798.6 & 61 & 3780.6 & 060.3 & 43 & 3230.5 & 1040.5 & 38 \\
\hline 6 & 4200 & 4282.7 & 816.8 & 67 & 4235.3 & 905.0 & 49 & 3810.8 & 969.7 & 68 \\
\hline 7 & 4700 & 4873.9 & 966.6 & 23 & 4704.1 & 1295.4 & 17 & 4006.1 & 1129.2 & 35 \\
\hline 8 & 5200 & 5145.6 & 830.6 & 46 & 4987.6 & 942.5 & 38 & 4651.4 & 1133.7 & 96 \\
\hline 9 & 5700 & 4875.7 & 796.7 & 14 & 5248.8 & 959.8 & 12 & 4792.4 & 1336.3 & 45 \\
\hline 10 & 6000 & 5773.0 & 781.8 & 27 & 5470.0 & 895.5 & 37 & 5464.8 & 1268.8 & 110 \\
\hline 11 & 6300 & 5907.8 & 606.5 & 9 & 5559.2 & 999.0 & 12 & 5247.1 & 1325.8 & 45 \\
\hline 12 & 6600 & 6386.7 & 738.1 & 18 & 5813.6 & 868.5 & 32 & 6031.7 & 1228.6 & 149 \\
\hline
\end{tabular}

*Promedios de diferencias de peso entre nacimiento y edad que se indica.

Comparando las columnas de promedio de aumento de peso de los tres tipos de leche se pueden observar las diferencias claras favorables a los niños que reciben leche materna. Los promedios del grupo amamantado se mantienen por encima de los otros. En relación al incremento teórico esperado, la diferencia también es favorable a los alimentados al pecho, hasta el $7 .^{\circ}$ mes.

En el Gráfico 3 se presentan los promedios observados y teóricos y, con el fin de analizar las tendencias, se ha estimado por método gráfico, atendiendo a la tendencia general de las curvas para leche materna $y$ artificial, los promedios para los meses $7 .^{\circ}$, $9 .^{\circ}$ y $11 .^{\circ}$. En estos meses loa valores originales pueden considerarge erráticos debido al bajo número de niños que acuden a control esos meses.

Si bien las diferencias son evidentes, en especial entre los niños alimentados con leche materna exclusiva y los que reciben leche artificial, es indispensable cuantificar la diferencia en forma estadística.

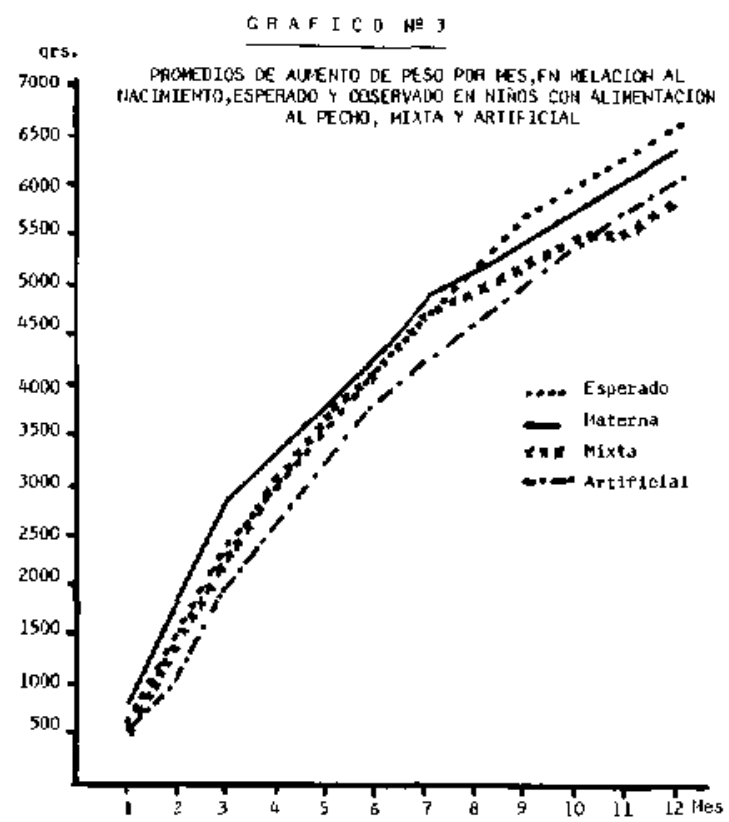


Se aplicó Ja prueba de $\mathrm{z}$ para diferencia entre prome$\operatorname{dios}^{6}$ y se fijó como nivel de significación el $5 \%$ ( $\mathrm{z}$ crítico al $5 \%$ unilateral $=1.65$ ).

\section{Tabla N." 4}

Valores de $z$ observado para comparar promedios de incremento de peso entre niños alimentados a pecho versus niños que reciben leche artificial total o parcialmente

\begin{tabular}{cccc}
\hline $\begin{array}{c}\text { Edad } \\
(1)\end{array}$ & $\begin{array}{c}\text { Natural vs. } \\
\text { artificial } \\
(2)\end{array}$ & $\begin{array}{c}\text { Natural vs. } \\
\text { mixta } \\
(3)\end{array}$ & $\begin{array}{c}\text { Artificial } \\
\text { v8. mixta } \\
(4)\end{array}$ \\
\hline 1 & $2.45^{*}$ & $4.13^{*}$ & -0.30 \\
2 & $5.53^{*}$ & $3 .(0)^{*}$ & $2.32^{*}$ \\
3 & $4.62^{*}$ & $2.57^{*}$ & $2.04^{*}$ \\
4 & $4.64^{*}$ & $2.30^{*}$ & $2.41^{*}$ \\
5 & $3.24^{*}$ & 0.32 & $2.60^{*}$ \\
6 & $3.06^{*}$ & 0.29 & $2.40^{*}$ \\
8 & $2.64^{*}$ & 0.82 & 1.62 \\
10 & 1.21 & 1.41 & 0.02 \\
12 & 1.20 & $2.36^{*}$ & -0.95 \\
- & & &
\end{tabular}

*Estadísticamente significativa.

Se puede afirmar, con un $95 \%$ de seguridad, que los niños que reciben leche matema presentan un incremento significativamente mayor hasta los 8 meses de vida, comparados con niños que reciben sólo leche artificial. A partir de los 10 meses los resultados no llegaron al nivel de significación estadística pero si puede considerarse que tienen una significación práctica.

Los beneficios de la lactancia materna exclusiva también se manifiestan, aunque en menor nivel, cuando ésta acompaña a la leche artificial (lactancia mixta). Como puede observarge, los resultados de la comparación de los promedios de incremento (Col.
3) son estadísticamente significativos en los cuatro primeros meses de vida. Hasta $\operatorname{los} 8$ meses los incrementos son muy semejantes en ambos grupos para luego ser notoriamente superior en los niños con lactancia materna exclusiva, a partir del $8 .^{\circ}$ mes la sitwación se invierte, los niños no alcanzan en promedio los incrementos esperados, por el contrario, se alejan. No hay que olvidar que son los meses $8 .^{\circ}, 10 .^{\circ}$ y $12 .{ }^{a}$ log realmente válidos, ya que en los meses $7 .{ }^{\circ}$, $9 .^{\circ}$ y $11.0^{\circ}$ no hay control por citación y la asistencia está condicionada en gran parte por factores que se pueden considerar negativos: enfermedad o desnutrición.

Creemos útil analizar los promedios de aumento de peso entre los niños alimentados al pecho con los incrementos teóricos esperados (Percentil 50 de Stuart). Con este objeto se calculó la prueba de $\mathrm{t}^{6}$ y los resultados mostraron que la leche materna exclusiva no sólo permite, en promedio, que los niños alcancen los niveles deseados sino que además entre el nacimiento y hasta los 5 meses, éstos ge sulperan en forma significativa. Entre $\operatorname{los} 6$ y 7 mesee el incremento no es significativo pero se mantiene por sobre lo esperado.

El grupo alimentado en forma mixta que combina leche materna con artificial no es fácil homologarlo en forma definitiva a los que sólo son amamantados o a los que sólo reciben mamadera. Algunos pediatras consideran que la introducción de la mamadera agrega los riesgos perjudiciales de ésta. Sin embargo, los resultados que se observaron en este estudio parecen indicar bajo el punto de vista de aumento de pego, que no es tan negativo como dar sólo mamadera. Los incrementos de peso son mayores entre los niños con alimentación mixta que entre los que sôlo reciben leche artificial. (Gráfico 3).

En la Col. 4 de la Tabla 4 aparecen los resultados

Tabla N.・ 5

Resultados prueba a para comparación promedio ineremento pesa niños con lactancia materna total o parcial y lactancia artificial; natural y artificial total o parcial

\begin{tabular}{|c|c|c|c|c|c|c|}
\hline \multirow{2}{*}{ Edad } & \multicolumn{2}{|c|}{$X$ incremento de pego } & \multirow{2}{*}{$\mathbf{z}$} & \multicolumn{2}{|c|}{$X$ incremento de peso } & \multirow{2}{*}{$\mathbf{z}$} \\
\hline & Nat. + Mixta & Artificial & & Nalura] & Mixta+Artif. & \\
\hline (1) & (2) & (3) & (4) & & & \\
\hline 1 & 730.5 & 513.3 & $1.95^{*}$ & 779.1 & 482.0 & $4.53^{*}$ \\
\hline 2 & 1709.1 & 1067.0 & $4.95^{*}$ & 1770.9 & 1305.0 & $5.26 *$ \\
\hline 3 & 2493.1 & 1922.3 & $3.92^{*}$ & 2587.6 & 2175.0 & $4.06 *$ \\
\hline 4 & 3202.0 & 2634.6 & $4.18^{*}$ & 3314.8 & 2878.2 & 3.901* \\
\hline 5 & 3811.2 & 3230.5 & $3.45^{*}$ & 3832.7 & 3522.5 & $2.06^{*}$ \\
\hline 6 & 4262.7 & 3810.8 & $3.29^{*}$ & 4282.7 & 3988.5 & $2.14^{*}$ \\
\hline $\mathbf{8}$ & 5074.2 & 4651.4 & $2.76^{*}$ & 5145.6 & 4747.0 & $2.27^{*}$ \\
\hline 10 & 5597.8 & 5464.8 & 0.75 & 5773.0 & 5466.1 & 1.29 \\
\hline 12 & 6019.9 & 6031.7 & -0.06 & 63886.7 & 5998.1 & 1.39 \\
\hline
\end{tabular}

2 crítico para nivel de signifieación $5 \%=1.64$.

*Estadísticamente significativo. 
de la prueba de z que compara los incrementos de peso entre niños que reciben alimentación láctea mixta y sólo mamadera. Hasta los 6 meses se observa una clara ventaja de la alimentación mìxta, las diferencias son estadísticamente significativas. $A$ los 9 meses los incrementos de peso son mayores en el grupo con lactencia mixta pero no alcanzan a niveles de significación. A partir de los 10 meses, el grupo con lactancia artificial presenta un incremento mayor de peso.

Otra forma de análisis es agregar los niñoz con alimentación mixta al grupo con leche matema exclusiva y comparar los nuevos promedios de incremento con los obtenidos por el grupo que sólo recibe mamadera o bjen agregarlos al grupo alimentado con leche artificial y compararlas con los niños que éólo son amamantados.
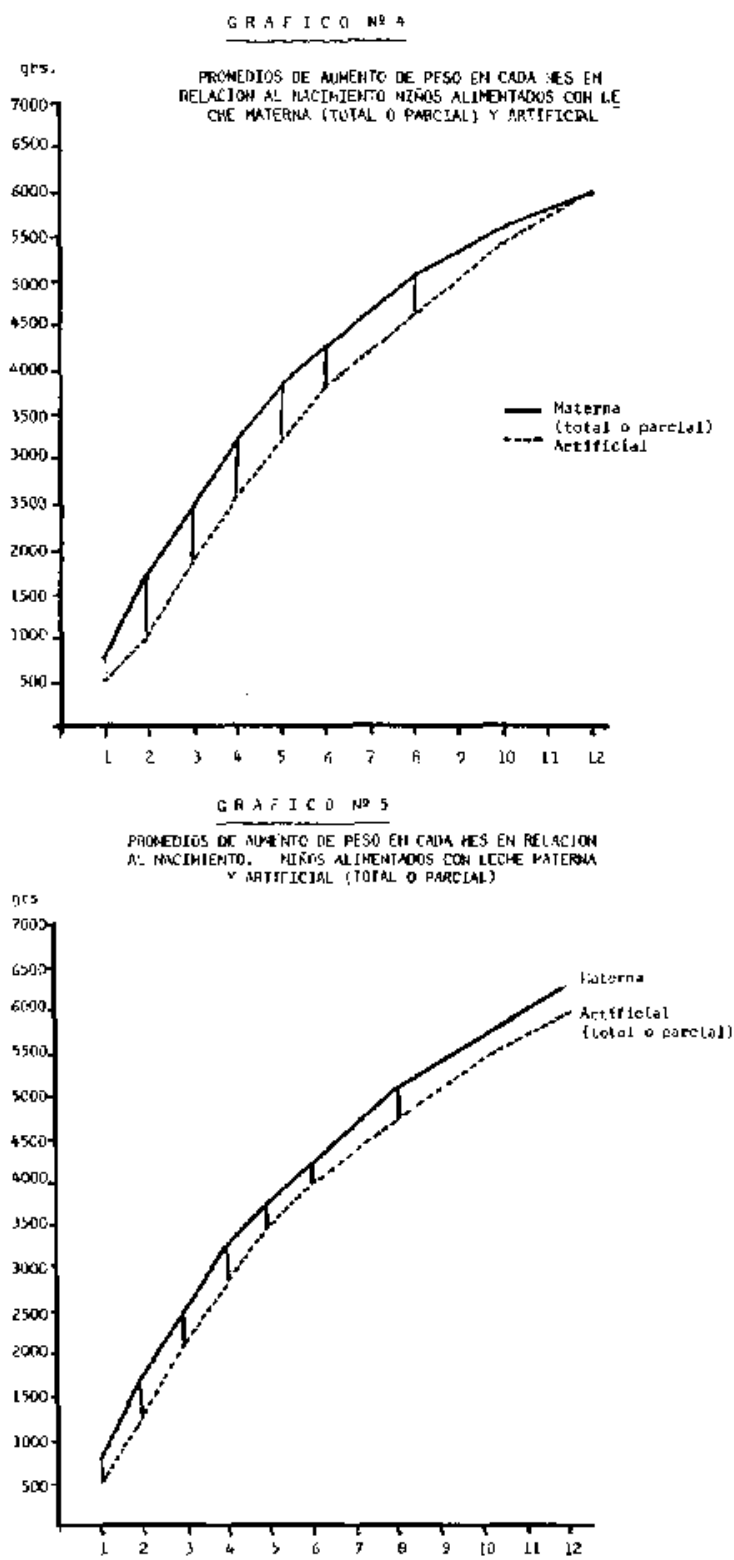

Al comparar loe promedios de incremento mensuales de peso del grupo que recibe leche materna absoluta o complementada con mamadera, con los de aquellos niños que reciben mamadera solamente, las ventajas de los primeros, con respecto a los segundos, son muy claras. Las diferencias son estadisticamente significativas hasta $\operatorname{los} 8$ meses. Los valores de $z$ son siempre mayores de 1.64 que es el z crítico para un nivel de significación del $5 \%$ en pruebas unilaterales. Entre los 9 y 11 meses las diferencias se hacen menores para terminar a los 12 meses prácticamente iguales, en los que reciben leche materna aunque sea en forma parcial.

Al integrar el grupo de lactancia mixta a los que son alimentados con leche artificial y comparar los promedios de incremento de peso conjunto de ambos grupos con los alimentados al pecho se observan diferencias estadísticamente significativas hasta $\log 8$ meses. Esto significa que los incrementos de peso del grupo de los niños con alimentación mixta no logran elevar los promedios de aumento de los que reciben sólo mamadera, lo que es explicable ya que estos últimos muestran niveles muy bajos de aumento de peso.

En resumen, el aumento de peso de un menor de un año varía significativamente según el tjpo de alimentación láctea que recibe. Hasta los 8 meses los niños amamantados muestran ganancias por sobre lo esperado o considerado como aumento ideal. Esta situación difjere de los niños que reciben alimentación mixta y más aún de los que sólo reciben mamadera. A partir de los 8 meses los nīnos estudiados muestran incrementos de pego por debajo de los perados, sin embargo, los niveles difieren en cada grupo de niño, según tipo de leche, manteniéndose el más alto aumento para los que reciben leche materna.

Al homologar el grupo con alimentación mixta a la materna absoluta los promedios de aumento mantienen diferencias estadísticamente significativas.

Los que sólo reciben mamadera no logran elevar los promedios de aumento de peso ni aún incluyendo entre ellos a los que reciben alimentación mixta. En este caso los promedios son estadjsticamente signifcativos más bajos que los observados entre los nin̄os amamantados.

\section{CONCLUSIONES}

1. A pesar de los esfuerzos no se ha logrado mumentar en forma significativa el período medio de lactancia materna exclusiva.

2. Los niños que nacen con mayor peso se mantienen al pecho por un período máe largo.

3. El aumento de peso durante el primer año es mayor si el niño recibe leche materna, sea éata en forma exclusiva o complementada con leche artificial. 


\section{RESUMEN}

Esta es la primera parte de un estudio en que se siguieron 207 niños desde el nacimiento basta los 12 meses. Se trata de mostrar que la leche materna contribuye positivamente al aumento de peso y de estaturs y reduce las diarreas y desnutrición durante el primer año.

Los niños pertenecen a un grupo socioeconómico bajo y se controlan en un consultorio del S.N.S.

El 50\% recibió leche materna por algo más de 3 meses. Poco más de un tercio hasta los 6 meses sólo un $15 \%$ hasta los 10 y $9 \%$ alcanza al año. Si se considera pecho más leche artificial el $50 \%$ alcanza $\log 8$ meses; $36 \%$ a los 10 meses y un $25 \%$ a los 12 meses.

Al considerar el peso al nacimiento se obaervan diferencias importantes. La mitad de los que pesaron entre 2500 y 3490 qr fueron alimentados al pecho sólo 3 meses y medio. Mientras que igual proporción de los que pesaron entre 3500 y $4190 \mathrm{gr}$ alcanzaron a $\log 5$ meses.

E] aumento de peso para los niños alimentados al pecho fue significativamente mayor que los que recibieron mamadera, hasta los 9 meses. Sin embargo, para todos los niños desde el $8 .^{\circ}$ mes el aumento es inferior que el esperado. ( $P_{50}$ de Stuart).

\section{HEFERENCLAS}

Meneghello, J. Undurraga, O. Ramos Galodn, R. "Pediatria" Tamo I. Ed, Interunódica. 1972 "Alimentación del Niño" p: 152153. "Enfermededea de la nutricion" p: 311.

2Potrl, A.; Sepultieda, "Leclencin materna", flev. Pediatria, Vol. 21. Oct.-Dic. 78 N. ${ }^{\circ} 4$ pp. $291-285$.

${ }^{3}$ Lopez, J.; Aloarado, E. Arcuch, S.; Cabiol, C; Alvera, E. "Laciancia matema y alounos factores condicionales". Cuadernos Mético-Soeinles. Col. XV. N.`4, Dic. 1974.

4 Incrementos pondoestaturales menswales y acumulados según Tabla de Stuart. Pub. Doc. 1125/78. Depto. de Nutricion. Fac. Med. Srgo. Norte, U. de Chile.

5Conzdlez Soto, G. "Crecinienlo y deastrolb del Niño". Rev, U. de Chile. N. ${ }^{\circ}$ 145. Pat 6. Finero 1979.

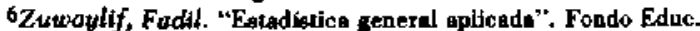
Interamer. S.A.

'Zaftartu, J. "Lactencia Materna y Nutrición Infantil", Rev. Med. de Chile. Vol. 102. N."11. Nov, 1974, pp. $892-885$.

BAriztia, A. "Diarreas y desnutrición infantiles". Rev. Chil. de Pediatria Ano. XlחI. N.*34, Marzo-Abril 1972, pp. 31-35.

${ }^{9}$ Marin, $P$. "Lactanein natural, desalio en enfermedad, denoutri ción y mortalidad infantil". Rer. Chilena de Pediatria. Vod. 45. N.'3. 1974. p: 259.

10Normas de Pediatrie N. 1. Acciones de Fomento y Protección. Miniaterio de Salud. Unidad del Niño y del Adolencente. Chile 1976. 